\title{
C-C Bond Formation Using Nickel-Photoredox Catalysis
}

\section{Key words}

nickel catalysis

photoredox catalysis

C-H

functionalization

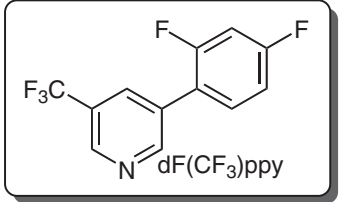

up to $85 \%$ yield

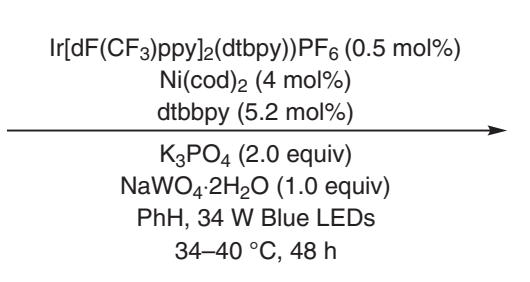

$\mathrm{R}^{1}=\mathrm{OAr}, \mathrm{Ar}, \mathrm{Cy}$

$R^{2}, R^{3}=$ various substituted alkyl or aryl moieties<smiles>CC(C)(C)c1ccc(C(=O)C2CCCCCCC2)cc1</smiles>

$55 \%$ yield<smiles>O=C1CC(C(=O)Oc2ccccc2)c2ccccc2O1</smiles>

$60 \%$ yield<smiles>CC(=O)CCC(C)C(=O)Oc1ccccc1</smiles>

$85 \%$ yield ( $\beta: \gamma: \delta 2.1: 5.4: 1)$<smiles>O=C(c1ccc(F)cc1)C1CCCCCCC1</smiles>

$62 \%$ yield<smiles>N#CCCC(C(=O)Oc1ccccc1)c1ccccc1</smiles>

$64 \%$ yield<smiles>CC(C)(CCN1C(=O)c2ccccc2C1=O)C(=O)Oc1ccccc1</smiles>

$57 \%$ yield ( $\alpha: \beta: \gamma: \delta 1: 3: 20.5: 4)$<smiles>CCCC(C)C(=O)Oc1ccccc1</smiles>

$68 \%$ yield $(\alpha: \beta: \gamma 1: 8: 4.6)$<smiles>O=C(c1ccccc1)C1CCCN1</smiles>

$55 \%$ yield<smiles>O=C1CCC(C(=O)Oc2ccccc2)C1</smiles><smiles>CC(C(=O)Oc1ccccc1)c1ccc(Oc2ccccc2)cc1</smiles>

$66 \%$ yield ( $\alpha: \beta 32: 1)$

$73 \%$ yield
Significance: The authors developed a nickelphotoredox-catalyzed $\mathrm{C}\left(\mathrm{sp}^{3}\right)-\mathrm{H}$ functionalization of a variety of unactivated alkyl or benzyl compounds using chloroformate derivatives. Mild coupling conditions allow the tolerance of several functional groups, leading to the corresponding carbonyl compounds in good yields.
Comment: From a mechanistic point of view, the authors hypothesized a catalytic cycle, which starts with an oxidative addition of chloroformate to a nickel(0) species. A SET forms a nickel(III) intermediate, which undergoes subsequent photoelimination, leading to a chlorine radical. Following $\mathrm{H}$-abstraction results in a carbon-centered radical. Addition of the nickel(II) complex and subsequent reductive elimination affords the carbonyl compound. 